\title{
Polarisation spontanée et interactions entre orbitales de Bloch au changement de phase para-ferroélectrique de $\mathrm{BaTiO}_{3}$ et $\mathrm{KNbO}_{3}$
}

\author{
L. Castet-Mejean \\ Laboratoire de Physique Electronique, Bât. 203, Université Lyon I, \\ 43, Bd du 11 Novembre 1918, 69622 Villeurbanne Cedex, France
}

(Reçu le 13 juin 1983, accepté le 15 septembre 1983)

\begin{abstract}
Résumé. - Les interactions entre orbitales de Bloch déterminées en prenant en compte les intégrales à trois centres et en introduisant certaines approximations permettent de décrire le changement de phase para-ferroélectrique des perovskites $\mathrm{BaTiO}_{3}$ et $\mathrm{KNbO}_{3}$ à partir des structures de bandes calculées par la méthode des liaisons fortes. Les effets des bords de bandes induits par la polarisation spontanée $\left(\boldsymbol{P}_{\mathrm{s}}\right)$ sont reproduits et estimés. Une interprétation de ces phénomènes au voisinage de la transition pour $T>T_{\mathrm{c}}$ associé à l'existence de $P_{\mathrm{s}}$ dans la phase cubique est proposée.
\end{abstract}

\begin{abstract}
The interactions between Bloch orbitals, determined by taking into account the threecentre integrals and by introducing some approximations, allow us to describe the para-ferroelectric phase change of $\mathrm{BaTiO}_{3}$ and $\mathrm{KNbO}_{3}$ perovskites from tight-binding band structures. The polarization induced band-edge effects are reproduced and estimated. An interpretation of these phenomena near the transition for $T>T_{\mathrm{c}}$ associated to precursor spontaneous polarization in the cubic phase is proposed.
\end{abstract}

Le changement de phase para-ferroélectrique des perovskites $\mathrm{BaTiO}_{3}$ et $\mathrm{KNbO}_{3}$ est étudié à partir de leur structure de bandes électroniques, déterminée par la méthode des liaisons fortes. Pour ces composés diélectriques et ferroélectriques, les interactions à courte distance et à grande distance sont équivalentes et pour suivre le changement de phase il est important de déterminer les interactions en prenant en compte les effets de l'environnement cristallin de façon assez représentative. Les calculs effectués dans la symétrie $D_{4 h}^{1}$ non ferroélectrique [1] et dans la symétrie $\mathrm{C}_{4 \mathrm{v}}^{1}$ ferroélectrique [2] reposent sur l'approximation de Wolfsberg-Helmoltz [3] et dans le cas de la symétrie $C_{4 v}^{1}$ en considérant seulement que les interactions entre orbitales atomiques varient proportionnellement aux recouvrements à partir de la phase cubique. Les présents calculs sont établis en introduisant les intégrales à trois centres pour exprimer les interactions. Cependant le calcul exact de ces intégrales est difficile et d'autre part, pour obtenir une expression assez simple des interactions permettant de suivre l'influence des facteurs les plus importants, des approximations ont été introduites. 
L'hamiltonien du cristal est écrit sous la forme habituelle :

$$
\hat{H}=T+\sum_{\mathbf{I}} V\left(\mathbf{r}-\mathbf{R}_{\mathrm{I}}\right)
$$

$V\left(\mathbf{r}-\mathbf{R}_{\mathrm{I}}\right)$ représente la contribution au potentiel cristallin total de l'atome localisé au site I, et $T$ est l'énergie cinétique. Les éléments de la matrice hamiltonienne expriment les interactions entre orbitales de Bloch :

$$
H_{l m}^{\mathrm{AB}}(\mathbf{k})=\sum_{\mathbf{R}_{\mathrm{AB}}} \exp \left(i \mathbf{k} \cdot \mathbf{R}_{\mathrm{AB}}\right)\left\langle\chi_{l}^{\mathbf{A}}(\mathbf{r})|\hat{H}| \chi_{m}^{\mathrm{B}}\left(\mathbf{r}-\mathbf{R}_{\mathrm{AB}}\right)\right\rangle
$$

$\chi_{l}^{\mathrm{A}}, \chi_{m}^{\mathrm{B}}$ désignent les orbitales atomiques de symétrie $l$ ou $m$ appartenant aux atomes $\mathrm{A}$ ou B dont la distance est $\mathbf{R}_{\mathrm{AB}}$. Les éléments matriciels de recouvrement $S_{l m}^{\mathrm{AB}}$ sont obtenus pour $[\hat{H}]=(1)$.

Les éléments de l'hamiltonien relatifs à une orbitale et à un site s'écrivent :

$$
\begin{aligned}
& H_{l l}^{\mathbf{A A}}=\left\langle\chi_{l}^{\mathbf{A}}(\mathbf{r})|\hat{H}| \chi_{l}^{\mathbf{A}}(\mathbf{r})\right\rangle \\
& =\left\langle\chi_{l}^{\hat{A}}(\mathbf{r})|T+V(\mathbf{r})| \chi_{l}^{\mathbf{A}}\right\rangle+\left\langle\chi_{l}^{\hat{A}(\mathbf{r})}\left|\sum_{\mathbf{l} \neq \mathbf{A}} V\left(\mathbf{r}-\mathbf{R}_{\mathbf{I}}\right)\right| \chi_{l}^{\mathbf{A}}(\mathbf{r})\right\rangle
\end{aligned}
$$

$\chi_{l}^{\mathrm{A}}$ sont les fonctions propres de l'hamiltonien de l'atome libre $[T+V(\mathbf{r})]$, avec les valeurs propres $\varepsilon_{l}^{\mathrm{A}}\left(q_{\mathrm{A}}\right)$ pour la charge atomique $q_{\mathrm{A}}$. En utilisant l'approximation des charges ponctuelles et les approximations classiques [4-6], le dernier terme peut être représenté par l'énergie de Madelung au site $\mathrm{A}$, et l'équation 3 s'écrit :

$$
H_{l l}^{\mathrm{AA}}=\varepsilon_{l}^{\mathrm{A}}\left(q_{\mathrm{A}}\right)+E_{\mathrm{Mad}}\left(q_{\mathrm{A}}\right) .
$$

Les éléments de l'hamiltonien relatifs à deux sites distincts peuvent se mettre sous la forme :

$$
H_{l m}^{\mathrm{AB}}\left(\mathbf{R}_{\mathrm{AB}}\right)=\left\langle\chi_{l}^{\mathrm{A}}(\mathbf{r})\left|T+V(\mathbf{r})+T+V\left(\mathbf{r}-\mathbf{R}_{\mathrm{B}}\right)-T+\sum_{\mathbf{I} \neq \mathrm{B}} V\left(\mathbf{r}-\mathbf{R}_{\mathrm{I}}\right)\right| \chi_{l}^{\mathrm{B}}\left(\mathbf{r}-\mathbf{R}_{\mathrm{AB}}\right)\right\rangle .
$$

Basch et al. [7] ont montré que les intégrales à deux centres, concernant les quatre premiers termes de l'hamiltonien, pouvaient être exprimées par la relation :

et d'autre part

$$
S_{l m}^{\mathbf{A B}}\left(\varepsilon_{l}^{\mathbf{A}}\left(q_{\mathbf{A}}\right)+\varepsilon_{m}^{\mathbf{B}}\left(q_{\mathbf{B}}\right)\right)
$$

$$
\cdot\left\langle\chi_{l}^{\mathrm{A}}(\mathbf{r})|T| \chi_{m}^{\mathrm{B}}\left(\mathbf{r}-\mathbf{R}_{\mathrm{AB}}\right)\right\rangle=T_{l m}^{\mathrm{AB}}
$$

représente l'intégrale à deux centres de l'énergie cinétique. En s'appuyant sur l'approximation des charges ponctuelles et une démonstration de Fenske et al. [6], les intégrales à trois centres relatives au dernier terme ont été transformées par Canadine et al. [5] :

$$
\sum_{\mathbf{I} \neq \mathrm{A}} q_{\mathrm{I}}\left\langle 1 / r_{\mathrm{I}} \mid \chi_{i}^{\mathrm{A}} \chi_{m}^{\mathbf{B}}\right\rangle \text {. }
$$

L'approximation de Mulliken [4, 8] analysée par différents auteurs [9-11] postulant un partage de la densité électronique sur les deux atomes suivant l'expression,

$$
\chi_{l}^{\mathbf{A}} \chi_{m}^{\mathbf{B}}=\frac{1}{2} S_{l m}^{\mathbf{A B}}\left(\chi_{l}^{\mathbf{A}} \chi_{l}^{\mathbf{A}}+\chi_{m}^{\mathbf{B}} \chi_{m}^{\mathbf{B}}\right),
$$

permet d'obtenir, en réintroduisant la relation 4 , une expression simplifiée des interactions entre orbitales atomiques :

$$
H_{l m}^{\mathrm{AB}}\left(\mathbf{R}_{\mathrm{AB}}\right)=F_{l m}^{\mathrm{AB}}\left[\frac{1}{2} S_{l m}^{\mathrm{AB}}\left(H_{l l}^{\mathrm{AA}}+H_{m m}^{\mathrm{BB}}+\varepsilon_{l}^{\mathrm{A}}+\varepsilon_{m}^{\mathrm{B}}+\frac{q_{\mathrm{A}}+q_{\mathrm{B}}}{R_{\mathrm{AB}}}\right)-T_{l m}^{\mathrm{AB}}\right]
$$


le facteur $F_{l m}^{\mathrm{AB}}$ est introduit pour permettre d'ajuster les résultats theoriques aux résultats expérimentaux. Les interactions ainsi déterminées ont permis de décrire les propriétés optiques, leurs modifications induites par le changement de phase para-ferroélectrique et les propriétés électrooptiques de $\mathrm{BaTiO}_{3}[12]$ et $\mathrm{KNbO}_{3}[13,14]$. Une formulation analogue a été proposée pour des calculs de clusters [15] mais les expressions des termes $H_{l l}^{\mathrm{AA}}$ sont différentes.

En introduisant seulement la variation des distances interatomiques apparaissant au changement de phase ce modèle rend compte de la polarisation spontanée $\left(\mathbf{P}_{\mathrm{s}}\right)$ d'origine ionique et électronique par l'intermédiaire des énergies de Madelung et des recouvrements. Les splittings les plus significatifs qui apparaissent au changement de phase sont localisés au bas de la bande de conduction (BC) en $\Gamma$ et $\Delta$ (Fig. 1). On peut exprimer l'écart $\Delta E$ entre les niveaux énergétiques des états $\Gamma_{5}$ ou $\Delta_{2}$ et l'état $\Gamma_{2}$, du minimum de BC en ne conservant que le terme de Bloch le plus important [14] associé aux interactions $2 \mathrm{p}$-nd entre l'oxygène axial $\mathrm{O}_{3}$ et l'atome central $\mathrm{B}$ du métal de transition :

$$
\Delta E=C(\mathbf{k})\left(\mathrm{pd} \pi_{3}-\mathrm{pd} \pi_{\overline{3}}\right)
$$

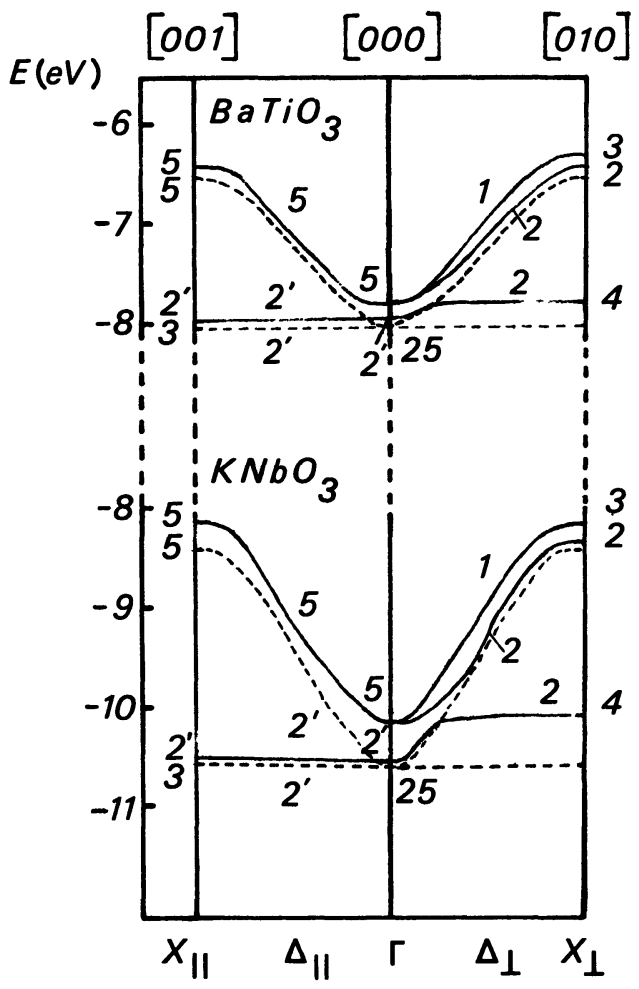

Fig. 1. - Etats $\Gamma$ et $\Delta$ de BC de plus faibles énergies : (-) phase tétragonale, (- -$)$ phase cubique. Les symboles sont ceux de B.S.W. [24] dans la phase cubique avec un indice supplémentaire pour la phase tétragonale, $/ /$ ou $\perp$, respectivement pour les vecteurs d'ondes parallèles ou perpendiculaires à $\mathbf{P}_{\mathbf{s}}$ [001]. Les chiffres qui caractérisent la symétrie des états sont les notations de B.S.W. et de Koster [25]. Les valeurs théoriques et expérimentales de $\Delta E$ dans la phase tétragonale sont respectivement : $160 \mathrm{meV}$ et $120 \mathrm{meV}$ pour $\mathrm{BaTiO}_{3}, 370 \mathrm{meV}$ et $240 \mathrm{meV}$ pour $\mathrm{KNbO}_{3}$.

[ $\Gamma$ et $\Delta$ CB states of lower energies : (-) tetragonal phase, $(--)$ cubic phase. The symbols are B.S. W's of the cubic phase [24] with a supplementary subscript for the tetragonal phase, $/ /$ ou $\perp$, respectively, for wave vectors parallel or perpendicular to $\mathbf{P}_{s}$ [001]. The numbers which specify the symmetry of the states are B.S.W. and Koster's notations [25]. $\Delta E$ theoretical and experimental values in the tetragonal phase are respectively : $160 \mathrm{meV}$ and $120 \mathrm{meV}$ for $\mathrm{BaTiO}_{3}, 370 \mathrm{meV}$ and $240 \mathrm{meV}$ for $\mathrm{KNbO}_{3}$.] 
les indices 3 et $\overline{3}$ désignent les distances parallèles à $P_{\mathrm{s}} \mathrm{B}-\mathrm{O}_{3}$ et $\mathrm{B}-\mathrm{O}_{\overline{3}}$, cette dernière étant la plus grande. Le coefficient $C(\mathbf{k})$ est obtenu à partir des coefficients $C_{i l}^{\mathrm{A}}$ du développement de l'orbitale du cristal de l'état $i$ considéré $\left(\Gamma_{5}\right.$ ou $\left.\Delta_{2 \perp}\right)$. Cette relation déduite des expressions 2 inclut les effets de la périodicité et se trouve simplifiée dans le plan $k_{z}=0$. Si l'on admet la loi quadratique introduite par Wemple [16] et Di Domenico [17] on a de plus la relation :

$$
\Delta E=\left(\beta_{11}-\beta_{12}\right) P_{\mathrm{s}}^{2} \simeq C(\mathbf{k})\left(\mathrm{pd} \pi_{3}-\mathrm{pd} \pi_{\overline{3}}\right)
$$

$\beta_{11}, \beta_{12}$ étant les potentiels de polarisation qui ont pu être déterminés précédemment [12, 13]. $P_{\mathrm{s}}$ peut être aussi estimé à partir d'une analyse de population $[12,13]$ mais l'expression 8 est pratique et plus explicite si l'on veut suivre l'évolution en fonction de la température. L'écart $\Delta E$ peut être considéré comme l'écart des gaps $G_{\mathrm{c}}-G_{\mathrm{a}} ; G_{\mathrm{c}}$ et $G_{\mathrm{a}}$ sont respectivement associés à une lumière polarisée parallèlement ou perpendiculairement à $\mathbf{P}_{\mathbf{s}}$. En effet les variations de la bande de valence sont faibles. En exprimant les interactions suivant la relation 6 on obtient :

$$
\begin{aligned}
\Delta E=F_{\mathrm{pd} \pi} C(\mathbf{k})\left[\frac{1}{2}\left(S_{\mathrm{pd} \pi_{3}}-S_{\mathrm{pd} \pi_{\overline{3}}}\right)\left(H_{\mathrm{pp}}^{\mathrm{O}_{3} \mathrm{O}_{3}}+H_{\mathrm{dd}}^{\mathrm{BB}}+\varepsilon_{\mathrm{p}}^{\mathrm{O}_{3}}+\varepsilon_{\mathrm{d}}^{\mathrm{O}_{3}}\right)+\right. \\
\left.\quad+\frac{1}{2}\left(S_{\mathrm{pd} \pi_{3}} \frac{q_{\mathrm{O}_{3}}+q_{\mathrm{B}}}{R_{\mathrm{O}_{3} \mathrm{~B}}}-S_{\mathrm{pd} \pi_{\overline{3}}} \frac{q_{\mathrm{O}_{3}}+q_{\mathrm{B}}}{R_{\mathrm{O}_{\overline{3}} \mathrm{~B}}}\right)-\left(T_{\mathrm{pd} \pi_{3}}-T_{\mathrm{pd} \pi \overline{3}}\right)\right] .
\end{aligned}
$$

Cette relation met en évidence le rôle joué par les principales grandeurs physiques qui déterminent les couplages associés à $P_{\mathrm{s}}$. L'effet dû aux recouvrements est faible, comparé à celui dû aux énergies cinétiques (voir tableau I), mais il est pondéré par les énergies de Madelung et d'ionisation qui amplifient l'effet minime qu'ils introduisent par les différences de Bloch. Les valeurs respectives des termes entre crochets sont pour $\mathrm{BaTiO}_{3}$ et à la température de $22{ }^{\circ} \mathrm{C}: 1,07 \mathrm{eV},-0,27 \mathrm{eV}$ et $-0,56 \mathrm{eV}$. Pour $\mathrm{KNbO}_{3}$ il serait préférable de prendre en compte l'effet dû à certains termes transversaux étant donné leur importance [14], néanmoins les expressions 8 et 9 sont encore représentatives.

Tableau I. - Paramètres des liaisons fortes des interactions $\mathrm{pd} \pi$ dans la phase tétragonale $\grave{a}$ $22{ }^{\circ} \mathrm{C}$ pour $\mathrm{BaTiO}_{3}$ et à $275{ }^{\circ} \mathrm{C}$ pour $\mathrm{KNbO}_{3} . F_{\mathrm{pd} \pi}\left(\mathrm{BaTiO}_{3}\right)=0,78$ et $F_{\mathrm{pd} \pi}\left(\mathrm{KNbO}_{3}\right)=0,62$.

[Tight-binding parameters of pd $\pi$ interactions in the tetragonal phase at $22{ }^{\circ} \mathrm{C}$ for $\mathrm{BaTiO}_{3}$ and at $275{ }^{\circ} \mathrm{C}$ for $\mathrm{KNbO}_{3} . F_{\mathrm{pd} \pi}\left(\mathrm{BaTiO}_{3}\right)=0.78$ and $F_{\mathrm{pd} \pi}\left(\mathrm{KNbO}_{3}\right)=0.62$.]

\begin{tabular}{|c|c|c|c|c|}
\hline Cristal & Distance & $S_{\mathrm{pd} \pi}$ & $T_{\mathrm{pd} \pi}(\mathrm{eV})$ & $\mathrm{pd} \pi(\mathrm{eV})$ \\
\hline $\mathrm{BaTiO}_{3}$ & $\mathrm{Ti}-\mathrm{O}_{3}$ & $-0,097$ & $-0,851$ & 2,415 \\
& $\mathrm{Ti}-\mathrm{O}_{\overline{3}}$ & $-0,058$ & $-0,286$ & 1,292 \\
$\mathrm{KNbO}_{3}$ & $\mathrm{Nb}-\mathrm{O}_{3}$ & $-0,151$ & $-1,437$ & 3,494 \\
& $\mathrm{Nb}-\mathrm{O}_{\overline{3}}$ & $-0,090$ & $-0,471$ & 1,918 \\
\hline
\end{tabular}

Les énergies des bandes de conduction $\Delta$ et plus particulièrement $\Delta_{2 \perp}$ augmentent au changement de phase para-ferroélectrique et elles jouent d'ailleurs un rôle déterminant dans les propriétés optiques ainsi que l'ont montré une analyse des moments de transitions interbandes [14] et les calculs des paramètres de Sellmeier où la translation de la position de l'oscillateur [12] est en accord avec les résultats expérimentaux de Johnson [18]. Les conclusions de Gähwiller [19] à partir de mesures de réflectivité, attribuant à un déplacement rigide des transitions vers les hautes énergies et indépendant du vecteur $\mathbf{k}$, les effets obtenus par l'action d'un champ électrique augmen$\operatorname{tant} \mathbf{P}_{\mathrm{s}}$ sont tout à fait conformes aux remarques précédentes. Mais en fait les bandes $\Delta$, outre une 
translation, subissent une contraction en ce qui concerne la largeur de bandes peu visible sur la figure 1, étant de faible amplitude.

Dans une matrice cubique en moyenne les effets sont semblables et peuvent être représentés par la relation 9. Les interactions à longue distance sont celles d'une maille cubique exprimées par les énergies de Madelung et la périodicité contenues dans cette relation. Les interactions à courte distance, dominées par les recouvrements et les énergies cinétiques, sont très sensibles aux variations locales des distances. Lors des vibrations du réseau il apparaît des couplages asymétriques et leurs effets sont pondérés comme il a été remarqué précédemment, et donc amplifiés par les énergies de Madelung. Les écarts à la linéarité du gap en fonction de la température, au-dessus du point de transition et à son voisinage [20] peuvent être interprétés par ce modèle. Quand la température diminue, la distance entre les atomes étant plus faible, les effets des couplages deviennent plus importants ; les énergies cinétiques $T_{\mathrm{pd} \pi}$ varient très rapidement en fonction de la distance ainsi que les recouvrements, de façon non linéaire en fonction de $1 / R$, et les termes correspondant dans la relation 9 ont une intensité suffisante pour que la position du bord de bande soit affectée. Les interactions entre orbitales de Bloch sont fonction de la périodicité et de la direction mais non du sens. Si le sens de $\mathbf{P}_{\mathrm{s}}$ est inversé dans le cluster voisin (îlot de $\boldsymbol{P}_{\mathrm{s}}$ ) ou au cours de la propagation de l'onde phononique, l'effet sur la structure de bande est toujours une augmentation de l'énergie du bas de BC. Ce phénomène s'établit dans les trois directions de l'espace et se manifeste par une augmentation de l'énergie des états $\Gamma$ et $\Delta$ par rapport à un réseau cubique non perturbé. La relation 9 permet d'exprimer et d'estimer cette augmentation qui n'est plus un splitting et qui est conforme aux résultats expérimentaux de Wemple [20] et permet de rendre compte de l'apparition de $\mathbf{P}_{\mathrm{s}}$ dans la phase cubique. Le couplage avec les vibrations du réseau est à l'origine de ce phénomène mais sa nature est néanmoins essentiellement électronique. La contribution de la polarisabilité électronique aux propriétés des ferroélectriques est souvent évoquée et notamment Slater [21] l'introduit dans l'expression de la constante diélectrique dans l'infrarouge précisant la nécessité de l'inclure dans l'étude de la transition de phase de ces matériaux. Ce modèle permet de prendre en compte cette polarisabilité, au niveau des orbitales, d'isoler et d'analyser ses effets notamment sur les phénomènes associés aux bords de bandes.

Dans l'hypothèse de fluctuations de la polarisation, d'îlots dynamiques de polarisation préexistant dans la phase cubique [20,22], ou en estimant un effet moyen à partir de la relation 9 on peut considérer une matrice cubique en moyenne, la $\mathbf{P}_{\mathrm{s}}$ étant alors déterminée essentiellement au niveau des orbitales atomiques et des orbitales du cristal, c'est-à-dire de nature principalement électronique. Certains phénomènes optiques au voisinage du point de transition et en particulier la déviation par rapport à la loi linéaire de l'indice de réfraction $n=f(T)$ au-dessus de la température de Curie [23] mise en évidence par les travaux récents de Burns et Dacol [22], peuvent être expliqués par les interactions entre orbitales de Bloch qui déterminent les augmentations des énergies des états de conduction et par l'analyse des forces d'oscillateur.

\section{Bibliographie}

[1] Michel-Calendini, F. M. and Mesnard, G., J. Phys. C. 6 (1973) 1709.

[2] Brews, J. R., Phys. Rev. Lett. 18 (1967) 662.

[3] Wolfsberg, M. and Helmolz, L., J. Chem. Phys. 20 (1952) 837.

[4] Mulliken, R. S., J. Chem. Phys. 46 (1949) 497.

[5] Canadine, R. M. and Hillier, I. H., J. Chem. Phys. 50 (1969) 2084.

[6] Fenske, R. F., Caulton, K. G., Radtke, D. D. and Sweeney, C. C., Inorg. Chem. 5 (1966) 951.

[7] BasCh, H., Ph. D. Thesis, Columbia University (1966). Basch, H. and Gray, H. B., Inorg. Chem. 6 (1967) 639.

[8] Rudenberg, K., J. Chem. Phys. 19 (1951) 1433.

[9] Dahl, J. D. and Balhausen, C. J., Adv. Quantum Chem. 4 (1968) 170.

[10] Shawitt, I. and Karplus, M., J. Chem. Phys. 38 (1963) 1256. 
[11] Companion, A. L. and Pan, R. G., J. Chem. Phys. 21 (1961) 2268.

[12] Castet-Mejean, L. and Michel-Calendini, F. M., Phys. Rev. B 23 (1981) 1.

[13] Castet-Mejean, L. and Michel-Calendini, F. M., Ferroelectrics 25 (1980) 475.

[14] To be publish and CAstet-Mejean, L., Ph. D. Thesis, Lyon I University (1982).

[15] JACQuiER, B., Ph. D. Thesis, Lyon I University (1975).

[16] Wemple, S. H. and Di Domenico, M., Applied Solid State Science (Academic Press) 1972.

[17] Di Domenico, M., Wemple, S. H. and Jr, J. Appl. Phys. 40 (1969) 720.

[18] Johnston, A. R., J. Appl. Phys. 42 (1971) 3501.

[19] GäHWiller, Ch., Solid State Commun. 5 (1967) 65.

[20] Wemple, S. H., Phys. Rev. B 2 (1970) 2679.

[21] Slater, J. C., Insulators, Semiconductors and Metals, Vol. III, p. 193 (New York Mc Graw-Hill).

[22] Burns, G. and DaCOL, F. H., Ferroelectrics 37 (1981) 661.

[23] Castet-Mejean, L., Solid State Commun., to be published.

[24] Boudaert, L. P., Smoluchowski, R. and Wigner, E., Phys. Rev. 50 (1936) 58.

[25] Koster, G. F., Solid State Phys. 5 (1957) 173. 